\title{
Intestinal Stoma Leak, CTCAE
}

National Cancer Institute

\section{Source}

National Cancer Institute. Intestinal Stoma Leak, CT CAE. NCI Thesaurus. Code C143592.

A finding of leakage of contents from an intestinal stoma (surgically created opening on the surface of the body). 